This file is a preprint and may contain errors or omissions not present in the final published version

\title{
Starting small: Exploring the origins of successor function knowledge
}

Rose M. Schneider, ${ }^{1}$ Ashlie Pankonin, ${ }^{1,2}$ Adena Schachner, ${ }^{1}$ David Barner ${ }^{1,3}$

${ }^{1}$ Department of Psychology, University of California, San Diego

${ }^{2}$ School of Speech, Language, and Hearing Sciences, San Diego State University

${ }^{3}$ Department of Linguistics, University of California, San Diego

Please address all correspondence to Rose M. Schneider, University of California San Diego, Department of Psychology, 9500 Gilman Drive, La Jolla, CA 92093. Email: roschnei@ucsd.edu 


\begin{abstract}
Although most U.S. children can accurately count sets by 4 years of age, many fail to understand the structural analogy between counting and number — that adding 1 to a set corresponds to counting up 1 word in the count list. While children are theorized to establish this Structure Mapping coincident with learning how counting is used to generate sets, they initially have an item-based understanding of this relationship, and can infer that, e.g, adding 1 to "five" is "six", while failing to infer that, e.g., adding 1 to "twenty-five" is "twenty-six" despite being able to recite these numbers when counting aloud. The item-specific nature of children's successes in reasoning about the relationship between changes in cardinality and the count list raises the possibility that such a Structure Mapping emerges later in development, and that this ability does not initially depend on learning to count. We test this hypothesis in two experiments and find evidence that children can perform item-based addition operations before they become competent counters. Even after children learn to count, we find that their ability to perform addition operations remains item-based and restricted to very small numbers, rather than drawing on generalized knowledge of how the count list represents number. We discuss how these early item-based associations between number words and sets might play a role in constructing a generalized Structure Mapping between counting and quantity.
\end{abstract}

Keywords: Number; counting; conceptual development; successor function 


\section{Introduction}

Humans are unparalleled in their ability to leverage relational structures in the construction of abstract concepts. Establishing commonalities between various component pieces of knowledge and forming analogical mappings over these structural alignments has grounded human innovation and discovery since antiquity (Hallyn, 2000). For example, Aristotle recruited letters of the alphabet to describe the properties and compositionality of atoms (Metaphysics, 985b4) by noting how atoms, like letters, have distinct properties and can be combined to form new structures. Roughly 2,000 years later, Kepler appealed to an analogy of light traveling from the sun in his proposal of a heliocentric model of the solar system (Gentner, Brem, Ferguson, \& Wolff, 1997). In each case, relational structures within a familiar domain (like the alphabet) are mapped onto another, less familiar domain (like atomic structure), to guide reasoning and discovery.

Analogical reasoning may not be limited to flashes of brilliance among geniuses instead, it may be a primary mechanism underlying children's more quotidian (but no less astonishing) conceptual development (Carey, 2009; Gentner, 2010; Xu, 2020). According to this idea, for young children as for Aristotle and Kepler, the construction of concepts from analogical mappings depends on learning about relational structures within different domains, and noticing how structures in one domain are similar to structures in another. For Aristotle and Kepler, this Structure Mapping (Gentner, 2010) process recruited extant relational structures; for children, however, these structures may be fragile or not yet acquired. This raises the question of how this process of discovering Structure Mappings begins in development. How do children transition from learning isolated facts about a domain of knowledge, to making abstract generalizations via analogy to another domain? 
Children's symbolic number acquisition provides a fertile case study for exploring the origin of Structure Mapping in conceptual development. Although children can recite a partial count list by around age 2 (Fuson, 1988), it takes them many years to discover how the structure of counting relates to number, and how counting up in the count list relates to adding items to a set - a type of analogical mapping. Multiple aspects of children's behavior provide evidence of this incomplete understanding. For instance, many two-year-olds fail to use counting to generate sets, and seem to regard the count list as a meaningless routine (similar to the $A B C$ s) rather than a powerful relational structure (Fuson, 1988). In addition, children's early comprehension of number words is item-based; that is, they learn the meaning of each number word separately, and serially in development. Thus, by around 2.5 years of age, many US children can generate sets of one without counting but provide random amounts when asked to generate a set of two. Some months later, children become "two-knowers," accurately generating sets of one and two, but not three; and then become "three-knowers" in the same way (Wynn, 1990, 1992).

At each "knower level" children do not use the count routine to generate either known or unknown numbers, and thus appear to lack a mapping between number and the structure of the count list. At around 4 years, however, these "subset-knowers" discover that counting can be used to generate any number. According to some accounts (e.g., see Carey, 2009 and Gentner, 2010), at this point children have acquired the "Cardinal Principle" (CP), meaning that they understand that the last word said while counting a set represents its cardinality (Gelman \& Gallistel, 1978).

How do children make this breakthrough? According to one influential account, children acquire the CP by establishing a Structure Mapping between the ordinal structure of the count list and the ordered set of cardinalities the count list represents (Carey, 2004; Gentner, 2010; 
Sarnecka \& Carey, 2008; for a review, see Marchand \& Barner, 2018). Specifically, children notice that the +1 positional differences between the numerals one, two, three, etc. is mirrored by +1 differences in cardinality, which leads them to hypothesize that this relationship holds for other numbers. This discovery and induction are proposed to allow children to count and construct sets of any size, by simply adding objects to a set as they count up the count list to a target number. According to Sarnecka and Carey (2008), this shift in children's understanding of the count list as a relational structure amounts to discovery of the successor function, a foundation of arithmetic described in formal systems like the Peano axioms (Peano, 1889), which states that for every number $n$, its successor is $n+1$. If a Structure Mapping of this kind underlies children's acquisition of the $\mathrm{CP}$, then children should only understand the successor relation between counting and cardinality once they have acquired the $\mathrm{CP}$.

To test this hypothesis, Sarnecka and Carey (2008) designed a paradigm known as the "Unit Task." In this task, children see some number of items added to a container while an experimenter says, "Look! There are $N$ items in the box!" The experimenter then adds 1 item to the box and asks, "Are there $N+1$ or $N+2$ items now?", e.g. are there 4 or 5 items. To provide the correct response, children must know that adding one item to an established cardinality corresponds to $\mathrm{a}+1$ increase in the count list (i.e., implicit successor knowledge), as shown in Figure 1a.

Sarnecka and Carey found that CP-knowers outperform subset-knowers on this task, in line with the idea that $\mathrm{CP}$ acquisition reflects a Structure Mapping between counting and number. However, in this and subsequent work, children's pattern of response is more complex than one would predict if this Structure Mapping were in place. For example, CP-knowers' advantage is limited to very small sets: Some young CP-knowers appear to know that adding +1 
to a set of four results in five, but lack a general understanding that, for any set $N$, adding +1 corresponds to counting up one word in the count list (i.e., $N+1$ ). What's more, some CPknowers fail even for the smallest numbers: Although chance on the Unit Task is $50 \%$, the CPknowers in Sarnecka and Carey (2008) had only 67\% accuracy for sets of 4 and 5. Similarly, other studies have found that many CP-knowers fail the Unit Task for these same numbers, despite being able to reliably produce them in their count list (Davidson, Eng, \& Barner, 2012; Spaepen et al., 2018). Lastly, CP-knowers who do succeed with numbers like 4 and 5 fail for even modestly larger numbers (such as 13), despite being able to count much higher (Davidson et al., 2012). Children exhibit item-specific performance for several years after CP acquisition, and do not demonstrate generalized successor knowledge until they have become exceptionally strong counters at around 6 years of age (Cheung, Rubenson, \& Barner, 2017; Schneider et al., 2020).

One conclusion that could be drawn from CP-knowers' item-specific Unit Task successes is that $\mathrm{CP}$ acquisition does not mark the point at which children establish a Structure Mapping between counting and cardinality. Instead, this limited ability to label the result of addition events for relatively small numbers could be based on a system of associative mappings between number words and sets, which are learned in a piece-meal fashion, separately for each number word (Sullivan \& Barner, 2014). For example, after experience observing sets of four being labeled with "four" and sets of five labeled with "five," upon being told that four objects are in a container, the child might form a visual representation of four items which is then updated when another item is added. Using an item-based associative mapping between this representation of five items and the word "five," the child could then report that there are five objects without appealing at any point to the count list's structure (Figure 1b). Given previous reports that 
associative mappings emerge sometime after children become CP-knowers, beginning with smaller numbers and slowly increasing up to around 6 (Sullivan \& Barner, 2014), this model might explain the limited advantage that CP-knowers initially have over subset-knowers.

a) Structure Mapping
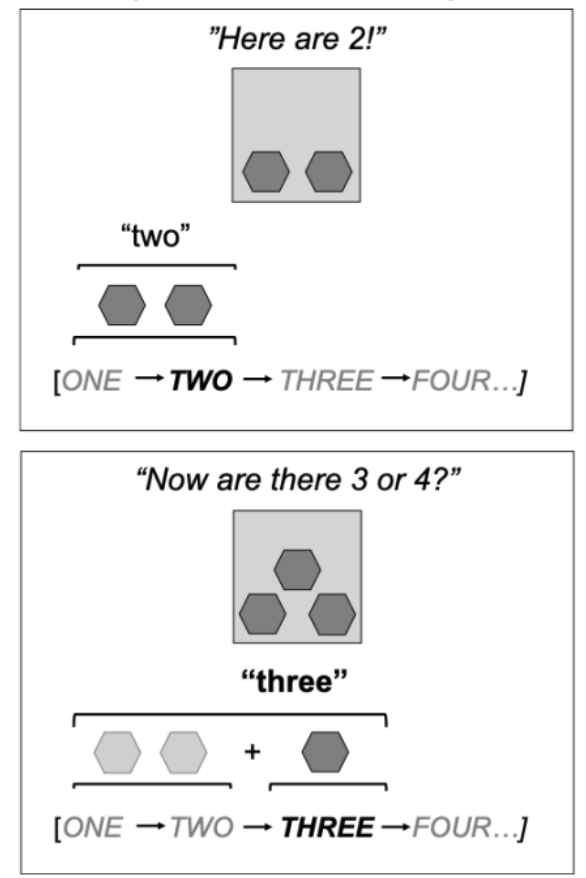

b) Associative Mapping

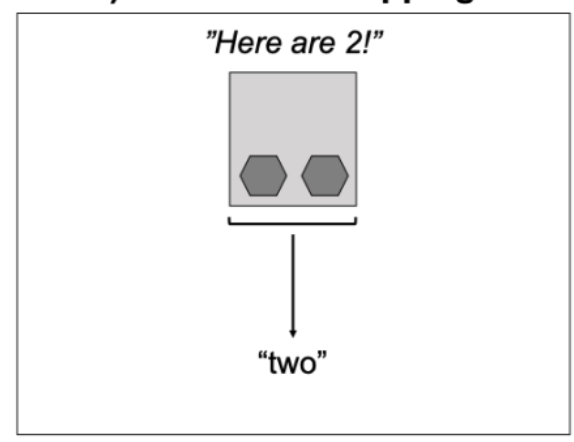

"Now are there 3 or 4 ?"

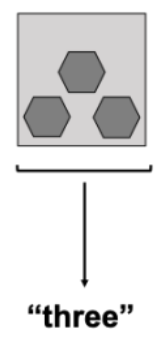

Figure 1. Representation of (a) Structure Mapping and (b) Associative Mapping mechanisms in the Unit Task. In (a), the result of Unit Task addition operations are labeled by reasoning about an isomorphism between these set operations and successor relations between number words in the count list. In (b), Unit Task addition operations are labeled by mapping known number labels directly onto set representations.

This Associative Mapping account makes several key predictions. First, children's ability to succeed in the Unit Task should be linked to their knowledge of particular number words, rather than to knowledge of the CP. As a result, all children - even subset-knowers - should be above chance in the Unit Task for known number words. The majority of prior work using the Unit Task has not been able to test for this possibility, however, since it has focused primarily on "large" numbers (such as 4, 5, and 6) which are unknown to subset-knowers.

The Associative Mapping account also makes a second prediction: Because children are proposed to succeed in the Unit Task by mapping number labels directly to set representations rather than reasoning about successor relations between number words in the count list - we 
should find only a weak relationship between Unit Task performance and count list knowledge. While CP-knowers eventually use knowledge of the count list's structure to acquire a generalized form of the successor function (Schneider et al., 2020), this alternative proposes that CP-knowers' item-specific knowledge may initially be independent of the count list. Likewise, we should find no association between knowledge of count list successor relations and Unit Task success in subset-knowers, as they do not yet understand the relation between the count list and cardinality.

Several pieces of evidence support the hypothesis that CP-knowers' ability to reason about Unit Task addition operations begins to take form much earlier in numerical development. Young children can perform the basic set operations necessary to succeed on the Unit Task even before they acquire the meanings of number words. At 5 months, infants can track the addition of 1 item to an occluded set of 1 , (Wynn, 1992a), and by 10 months can track the addition of 1 graham cracker to a bucket to discriminate between sets of 2 and 3 (Feigenson, Carey, \& Hauser, 2002; for a review of this literature, see Cantrell \& Smith, 2013). Similarly, children between the ages of 2.5 and 4 years can solve nonverbal addition problems with sets of 1-3 (Huttenlocher, Jordan, \& Levine, 1994). Finally, Hughes (1981) found that children as young as 3 years (who were likely subset-knowers) succeeded on a task similar to the Unit Task for small numbers (13). Together, these results suggest that even subset-knowers can not only perform the set operations required in the Unit Task, but can also map these set representations to number words.

These studies lend credibility to the Associative Mapping hypothesis. On this hypothesis, performance on the Unit Task may not have any special relationship to the $\mathrm{CP}$ transition: The ability of CP-knowers to succeed in the Unit Task may begin to take form when children are subset-knowers, and may simply be restricted to smaller numbers that are more familiar to 
younger children. However, currently, there is little direct evidence regarding this point. Some existing data, however, suggests this as a possibility: Although Sarnecka and Carey (2008) focused their analyses on performance for the numbers 4 and 5, data from pretest trials show that subset-knowers above chance for sets of 1 and 2, numbers within their known range.

Previous studies also leave open how children at different developmental stages might differ in the ways they compute addition operations - e.g., via Structure Mapping or Associative Mapping. Young CP-knowers may initially rely on Associative Mappings when performing the Unit Task, and only older, more experienced CP-knowers may recruit a full-fledged Structure Mapping between counting and cardinality. Becoming a CP-knower may be a necessary, but not sufficient, step in the construction of a Structure Mapping. On this view, subset-knowers may also acquire Associative Mappings early in development, which continue to develop after the CP transition.

Another possibility is that all CP-knowers have acquired a Structure Mapping between counting and number. Though past studies have generally reasoned that this would predict success at larger numbers, it remains possible that other factors limit CP-knowers' deployment of Structure Mapping - e.g., such as still-developing count list fluency (Chu et al., 2020; Schneider et al., 2020; Siegler \& Robinson, 1982), or the working memory challenge of tracking changes to sets while simultaneously reasoning about successor relations between still freshly-learned words. Compatible with this discontinuity hypothesis, Spaepen and colleagues (2018) provide evidence that CP-knowers' — but not subset-knowers' — Unit Task performance improves after count list training.

In the current work, we had two goals. First, our primary question was whether Unit Task performance was related to familiarity with specific number words, rather than CP knowledge. 
To investigate this, we tested when both subset-knowers and CP-knowers could label addition events in the Unit Task for a range of small numbers and asked whether their performance on specific numbers was related to their knower level (i.e., their set of known number words) such that even subset-knowers succeed for known numbers. Second, we sought to test the possible mechanisms underlying item-specific Unit Task performance, and whether subsetknowers and young CP-knowers both deploy Associative Mapping, or whether the transition to the CP stage also marks a transition to Structure Mapping. To do so, in Experiment 1, we explored the relationship between knowledge of number words, Unit Task performance, and general count list knowledge. In Experiment 2, we further tested the role of count list knowledge to investigate the mechanisms through which children may begin to establish this item-based ability.

\section{Experiment 1}

\section{Method}

The methods and analyses of this study were pre-registered prior to data collection. The pre-registration can be found at https://osf.io/deqzk/?view_only=e49622708a214438bb095f18bdaa8224. All methodological and analytical choices were as pre-registered, unless stated otherwise in-text.

Participants. We pre-registered a minimum $n$ of 68 participants, with $n=44$ subsetknowers and $n=24 \mathrm{CP}$-knowers, post-exclusions. This was pre-registered on the basis of a power analysis using pilot data, indicating that a sample of 44 could detect an effect size of $d=$ 0.5 with $90 \%$ power. Because children completed Give-N at the study's end, we did not preregister a specific number of $N$-knowers.

We recruited 89 typically-developing, English-speaking children between 2 and 4 years of age from preschools and museums in San Diego, California, USA and Comox Valley, British 
Columbia, Canada. Thirteen children were tested but excluded from analyses due to: failure to complete all trials of the Unit Task and Give-N $(n=8)$; lack of any number knowledge as determined by Give-N (i.e., non-knowers; $n=2)$; outside the age range $(n=2)$; or failure to comprehend tasks $(n=1)$. After these exclusions, our analyzable sample included 76 participants (Table 1).

\begin{tabular}{|c|c|c|c|c|c|}
\hline \multirow{5}{*}{ Subset-knowers } & $n$ & $M_{\text {age }}\left(S D_{\text {age }}\right)$ & & $n$ & $M_{\text {age }}\left(S D_{\text {age }}\right)$ \\
\hline & \multirow{4}{*}{$\begin{array}{c}52 \\
(n \text { female }=25 \\
n \text { male }=27)\end{array}$} & \multirow{4}{*}{$3.38(0.39)$} & 1-knowers & 18 & $3.12(0.42)$ \\
\hline & & & 2-knowers & 18 & $3.56(0.24)$ \\
\hline & & & 3-knowers & 14 & $3.43(0.35)$ \\
\hline & & & 4-knowers & 2 & $3.74(0.19)$ \\
\hline CP-knowers & $\begin{array}{c}24 \\
(n \text { female }=13 \\
n \text { male }=11)\end{array}$ & $3.6(0.30)$ & & & \\
\hline
\end{tabular}

Table 1. Demographic information by knower-level for Study 1 for subset- and CP-knowers (bottom). Demographic information for $\mathrm{N}$-knowers is reported on the right.

\section{Stimuli, design, and procedure}

Children were tested individually in a quiet spot within the classroom or museum. Participants received the tasks in a fixed order (Unit Task, Give-N, and Highest Count). Unit Task. We used a modified version of the Unit Task (Sarnecka \& Carey, 2008) to assess children's item-based knowledge. The experimenter presented children with an opaque container and some plastic fish, saying, "This is my fishbowl and these are my fish." The experimenter then placed between one and five fish in the container and said, "Look! $N$ fish are swimming in the fishbowl." The experimenter covered the container if children attempted to count. The experimenter then replaced the lid and asked, "How many fish are in the fishbowl?" Children were given two opportunities to respond; if they failed both, the experimenter told them how 
many fish were in the container. The experimenter then added one fish and asked "Are there $N+1$ or $N+2$ fish now?" Order of alternatives was counterbalanced across trials. If children failed to pick an alternative, the experimenter provided them again. Participants received a training trial with feedback in which one fish was added to an empty container, and then 10 test trials without feedback. The queried numbers (1, 2, 3, 4, and 5) were presented in a pseudo-randomized order. The correct response for a given $N$ was $N+1$. "I don't know" responses $(n=2 / 755)$ were coded as incorrect. If children did not respond, or provided a non-numeric response (other than "I don't know), the trial was excluded from analysis. Trials were classified as involving numbers either within or outside children's $N$-knower level (i.e., for a 2-knower, trials with a starting state of 1 and 2 items were classified as "within" the child's known number range, while 3, 4, and 5 were "outside" their range).

Give-N. We used a titrated version of Give-N (Wynn, 1990) to assess children's N-knower level. The experimenter provided children with 10 identical plastic objects (e.g., strawberries), and a plate. After familiarizing the child with the game the experimenter asked them to put $N$ items on the plate. When the child finished, the experimenter asked, "Is that $N$ ? Can you count to make sure?" If the child recognized an error, they were permitted to fix it. If the child succeeded in giving $N$, on the subsequent trial the experimenter asked for $N+1$, up to the number six. If the child failed to generate a set of $N$ the experimenter asked next for $N-1$. This pattern of titration continued until the child's knower level was identified.

Children were classified as $N$-knowers (e.g., 3-knower) if they correctly provided $N$ (e.g., three strawberries) on at least two out of the three trials that $N$ was requested and, when the child provided $N$, two-thirds of the time it was in response to a request for $N$. To be classified as a CPknower, children needed to correctly generate a set of six at least two out of three times when 
requested. Because we could not ensure our sample would have an even distribution of $\mathrm{N}$ knowers, our confirmatory analyses collapse over all non-CP-knowers as "subset-knowers."

Highest Count. We used the Highest Count task as a measure of counting experience beyond the $\mathrm{CP}$ stage, as well as to test whether, even in the subset stages, count list proficiency might enhance knowledge of successor relations between the number words involved in the Unit Task. For young children, this measure can reflect differences in number language exposure (LeFevre, Clarke, \& Stringer, 2002), and has been used to assess count list proficiency (Cheung et al., 2017; Chu et al., 2020; Davidson et al., 2012; Schneider et al., 2020). The experimenter prompted the child by saying, "I want you to count as high as you can! Can you start counting with one?" The child's Highest Count was the largest number counted to before an error (allowing for one error), or the point at which the child could not continue. If a child stopped counting, the experimenter prompted them once by saying "Do you know what comes next/after $N$ ?" If a child could not continue, the task was ended. Otherwise, the child was able to continue counting.

\section{Results}

Our primary question was whether children's Unit Task performance was related to their familiarity with specific number words, rather than knowledge of the CP. If so, we would predict that (a) CP-knowers would perform better on the Unit Task than subset-knowers (potentially because they are familiar with a larger number of specific number words), but crucially that (b) subset-knowers would perform above chance at trials involving known numbers.

First, consistent with previous work, a generalized linear mixed effects model (GLMM $\left.{ }^{1}\right)$ predicting Unit Task success from CP-knower status and age with a random effect of participant

\footnotetext{
${ }^{1}$ All GLMMs were fit in R using the 'Ime4' package (Bates, Martin, Maechler, Bolker, \& Walker, 2015).
} 
indicated a significant effect of CP knowledge $\left(\chi^{2}(1)=8.29, p=.004\right)$, with CP-knowers demonstrating greater Unit Task accuracy $(M=.70)$ in comparison to subset-knowers $(\beta=0.52$, $p=.004 ; M=.58)$, as shown in Figure 2. Age was not a significant predictor in this model $(\beta=$ $0.09, p=.29)$. Post hoc tests revealed that despite overall higher accuracy, CP-knowers were at chance for sets of $5(t(23)=-0.72, p=.48)$. While some previous studies report higher performance for CP-knowers on these small numbers, they tested older children than our current sample; in line with our findings, previous work finds that younger CP-knowers are often at chance for small numbers (Cheung et al., 2017; Davidson et al., 2012; Spaepen et al., 2018).

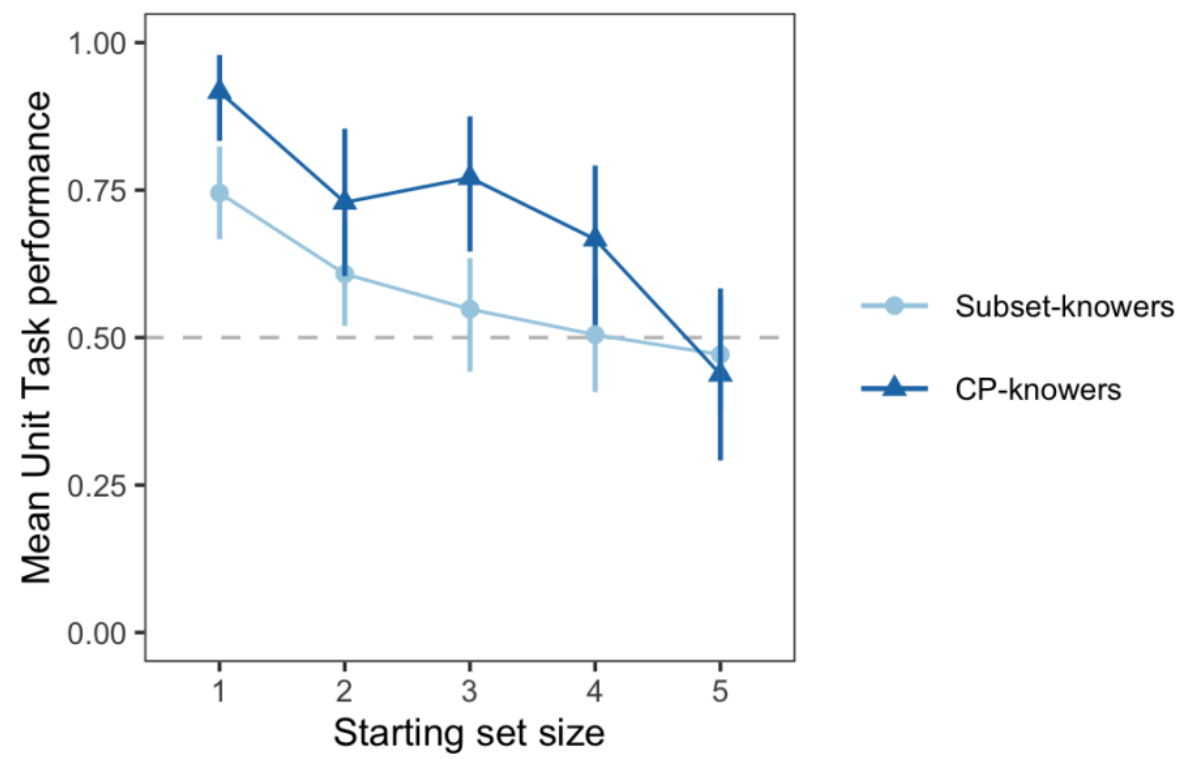

Figure 2. Mean Unit Task performance for each starting set size, grouped by CP-knower status. Error bars represent 95\% confidence intervals, computed by nonparametric bootstrap. Dashed line indicates chance performance (50\%).

We next turned to subset-knowers, exploring whether (despite lower performance in comparison to CP-knowers) they could label Unit Task addition events for known numbers. To test this, we built a null GLMM² with data from subset-knowers predicting Unit Task accuracy. This model indicated that subset-knowers were significantly above chance overall (Wald $Z=$

\footnotetext{
${ }^{2}$ Null models were constructed with the formula: Correct $\sim 1+(1 \mid$ Participant $)$.
} 
$3.38, p=.0007)$. A second model revealed that this effect was even stronger for trials in which the starting number was within subset-knowers' known range (Wald $Z=5.19, p<.0001$ ), with higher accuracy for known items $(M=0.70)$ in comparison to unknown items $(M=0.50)$. A follow-up analysis indicated that subset-knowers' performance for known numbers did not differ from CP-knowers' $(\beta=-0.06, p=.79)$.

Our classification of trials as within or beyond a child's knower level in the above analyses considered only the base addend and not the final sum (which allowed the inclusion of one-knowers). As a result, the results of some +1 computations exceeded a child's $N$-knower level. We thus conducted post hoc tests to explore whether subset-knowers' performance was greater on trials where the final result remained within their $N$-knower level. A third model indicated that this was indeed the case: Performance improved when the final sum, not just the starting set, was within children's knower level (Wald $Z=6.24, p<.0001 ; M=0.85$ ) Thus, although subset-knowers were less accurate on the Unit Task than CP-knowers overall, they were above-chance when the majority of numbers queried were plausibly within their known number range. Importantly, subset-knowers' performance indicates that item-based Unit Task success is not dependent on acquisition of the CP.

If such item-specific ability is not dependent on $\mathrm{CP}$ acquisition, then when does it emerge, and what is its relation to number word acquisition? As predicted by Associative Mapping, subset-knowers' Unit Task success is strongly related to their number word knowledge. We further explored this pattern in follow-up analyses testing $N$-knowers' mean performance for individual items against chance $(m u=.5)$. These analyses provide further evidence that subset-knowers' Unit Task performance tracked with their $N$-knower level (Figure 3). One-knowers were at chance for all items, as predicted, since all trials (even 1+1) returned a 
result outside of their known number range (all $p s>.50$ ). Two-knowers were above chance only for sets of $1(t(17)=6.65, p<.0001)$, and 3-knowers were above chance both for sets of $1(t(13)$ $=8.83, p<.0001)$ and $2(t(13)=3.80, p=.002)$. Four-knowers were not included in these analyses due to their small $n$. While these analyses are underpowered, they nevertheless support the indication of our confirmatory analyses that subset-knowers' Unit Task success is closely tied to their knowledge of specific number words, and that item-based learning begins almost as soon as children start to acquire the meanings of number words.
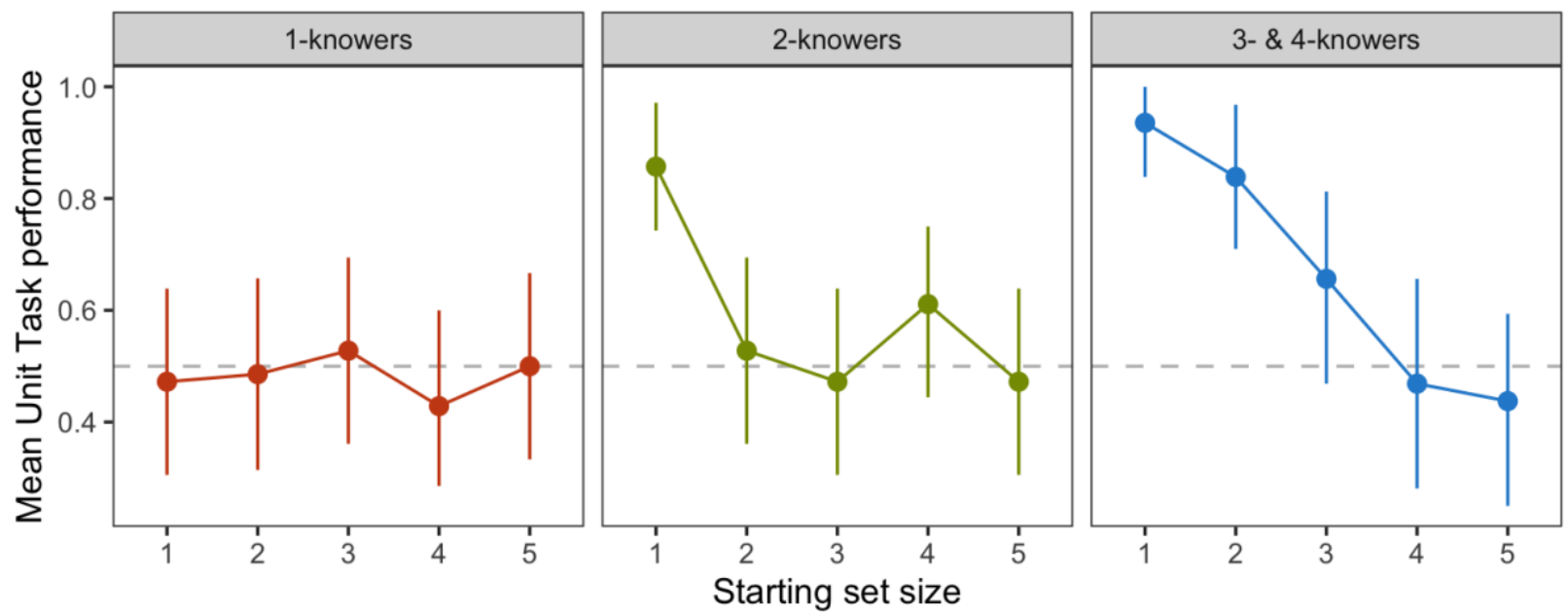

Figure 3. Mean Unit Task performance for all task items by $N$-knower level. Four-knowers are grouped together with 3-knowers due to small $n$ (2). Error bars indicate $95 \%$ confidence intervals, computed by nonparametric bootstrap. Dashed line indicates chance performance (50\%).

We next explored whether, even in the subset-knower stage, this item-specific knowledge could be explained by Structure Mapping. We did this by exploring the relationship between the Unit Task and Highest Count. Our logic in this analysis was that if children succeed on the Unit Task through a Structure Mapping between count list successor relations and set operations, then children with increased knowledge of these successor relations (as indicated by greater Highest Counts) should demonstrate greater Unit Task accuracy. We used Likelihood Ratio Tests to determine whether the addition of a Highest Count term explained additional variance in a 
GLMM predicting Unit Task from age for CP and subset-knowers. ${ }^{3}$ This analysis indicated that Highest Count was not predictive of Unit Task performance for subset-knowers $\left(\chi^{2}(1)=0.41, p=\right.$ $0.52)$ or CP-knowers $\left(\chi^{2}(1)=1.60, p=.21\right)$. This provides preliminary evidence that changes in count list knowledge do not alone explain changes in Unit Task performance during this developmental window.

\section{Discussion}

In Experiment 1, we investigated the emergence of item-specific Unit Task success in $\mathrm{CP}$ - and subset-knowers. Previous work has argued that $\mathrm{CP}$ acquisition reflects a mapping between the structure of counting and cardinality and that unlike subset-knowers, CP-knowers understand that adding items to a set corresponds to counting up in the count list, and vice versa. CP-knowers' greater accuracy on the Unit Task (a task which measures implicit knowledge of this cardinality-count list mapping) in comparison to subset-knowers' has been offered in support of this hypothesis.

In Experiment 1, we found several pieces of evidence against this account. First, we found that children succeed on the Unit Task well before they have acquired the CP: Subsetknowers performed above chance for numbers within their knower level. This suggests that the ability to label the result of addition operations is learned through a continuous process, which begins when children first start to learn the meanings of number words, rather than only after $\mathrm{CP}$ acquisition. Second, while CP-knowers outperformed subset-knowers, this advantage was limited and did not extend to the largest number tested (i.e., 5), suggesting that their advantage was one of degree, rather than qualitative in nature. Finally, unlike work in older children which shows a strong relationship between count list familiarity and generalized successor knowledge

\footnotetext{
${ }^{3}$ Because CP-knowers had significantly greater Unit Task performance than subset-knowers, we conducted these analyses separately for the two groups.
} 
(Cheung et al., 2017; Schneider et al., 2020), we did not find that counting proficiency was related to either subset- or CP-knowers' item-based Unit Task success. Together, these results suggest that previously reported differences between subset and CP-knowers on the Unit Task are likely attributable to familiarity with the specific numbers queried, rather than qualitative differences in underlying mechanisms.

One key limitation remains in Experiment 1: The Highest Count Task, while a good proxy for general exposure to counting, may not be the best way to test children's knowledge of count list successor relations. Thus, by extension, this measure may not be sensitive to whether children use a Structure Mapping made between these relations and Unit Task set operations. First, much of the variability in the Highest Count measure in our study occurred well beyond the small number range, making it a poor test of differences in familiarity with smaller numbers. Second, the ability to count does not necessarily require a recognition that counting is a structured, relational system. Initially, children recite numbers as an unbroken chain, only later recognizing that numbers are separate entities, but still relying on a memorized and unstructured list for several years (Chu et al., 2020; Fuson, 1988; Schneider et al., 2020). Given this, subsetknowers and CP-knowers who count to similar numbers may nevertheless represent the count list differently, and deploy it differently during the Unit Task.

For these reasons, in Experiment 2 we measured children's ability to reason about successor relations between numbers in the count list using the Next Number task, which asks children to count up from arbitrary numbers (e.g., “four, what comes next?”). On the Structure Mapping hypothesis, performance on the Unit Task involves mappings relations between number words to relations between sets. By this account, children who see a set labeled as "four" and watch one item added are hypothesized to reason that adding 1 item to the set motivates 
counting up one word in the count list, from "four" to "five." Therefore, any child who deploys this mechanism should be able to easily identify the successor of implicated numbers - e.g., that the number after "four" in the count list is "five" - and thus succeed at the Next Number task. Given this logic, we reasoned that if children use Structure Mapping during the Unit Task, then either their Next Number performance should exceed Unit Task performance, or performance on the two tasks should not differ in terms of accuracy. Although we might expect lower Next Number performance in comparison to the Unit Task due to response format (free response vs. two-alternative forced-choice), we address this issue by exploring whether performance on these tasks differs as a function of CP-knower status, which would suggest a difference in numerical knowledge, rather than response format. Previous work finds a strong correlation between these two tasks among older CP-knowers, and this correlation remains when both tasks use an openended response format (Schneider, Sullivan, Guo, \& Barner, in press).

In contrast, the ability to reason about count list successor relations is not required by Associative Mapping: By this account, children update set representations, and then map these updated representations directly to associated number words. If children use Associative Mapping via direct associations without appeal to count list knowledge in the Unit Task, we would expect a weak or absent relationship between the Next Number and Unit Task. Additionally, this account would predict it is possible for children to succeed on the Unit Task prior to Next Number; for example, children may be able to label the result of adding one item to a set of one in the Unit Task despite being unable to say what comes after "two" in the count list.

\section{Experiment 2}

\section{Method}


The methods and analyses of this study were pre-registered prior to any data collection. The preregistration can be found at

https://osf.io/deqzk/?view only=e49622708a214438bb095f18bdaa8224. All methodological and analytical choices were as pre-registered, unless stated otherwise in-text.

Participants. We pre-registered a minimum $n$ of 68 participants, with $n=44$ subset-knowers, post-exclusions, and $n=24 \mathrm{CP}$-knowers, post-exclusions. We again attempted to recruit children from all knower levels.

We recruited 107 typically-developing English-speaking children between the ages of 2 and 4 years of age from local preschools, museums, and the surrounding community in San Diego, California, USA. Of these, 28 children were tested but excluded from analyses as preregistered due to: failure to complete the minimum number of trials in Give-N and the Unit Task $(n=16)$; lack of any number knowledge (i.e., 0 -knowers, $n=4)$; outside of age range $(n=4)$; experimenter error $(n=2)$; and non-English primary language $(n=1)$. After these exclusions, our final analyzable sample included 73 children $(n$ female $=41)$, shown in Table 2 .

\begin{tabular}{|c|c|c|c|c|c|}
\hline \multirow{6}{*}{ Subset-knowers } & $n$ & $M_{\text {age }}\left(S D_{\text {age }}\right)$ & & $n$ & $M_{\text {age }}\left(S D_{\text {age }}\right)$ \\
\hline & \multirow{5}{*}{$\begin{array}{c}45 \\
(n \text { female }=26 \\
n \text { male }=19)\end{array}$} & \multirow{5}{*}{$3.13(0.50)$} & 1-knowers & 14 & $2.98(0.49)$ \\
\hline & & & 2-knowers & 18 & $3.23(0.45)$ \\
\hline & & & 3-knowers & 8 & $2.84(0.47)$ \\
\hline & & & 4-knowers & 4 & $3.56(0.36)$ \\
\hline & & & 5-knowers & 1 & 3.92 \\
\hline CP-knowers & $\begin{array}{c}28 \\
(n \text { female }=15 \\
n \text { male }=13)\end{array}$ & $3.69(0.31)$ & & & \\
\hline
\end{tabular}


Table 2. Demographic information by knower-level for Study 2 for subset- and CP-knowers (bottom). Demographic information for $\mathrm{N}$-knowers is reported on the right.

Stimuli, design, and procedure. Stimuli and methods were identical to Experiment 1 with two exceptions. First, as mentioned above, we tested children's knowledge of count list successor relations with the Next Number task. Second, to control for the possibility that children succeeded on the Unit Task through subitizing the final set, we inserted fish into the bowl through a lid with a small slot (rather than removing the lid; this prevented subitizing by preventing children from viewing all items in the set at once). Participants received the tasks in a fixed order (Unit Task, Next Number, Give-N, and Highest Count).

Next Number Task. The experimenter introduced the task by saying, "Now we're going to play a game where I say a number and you tell me what number comes next. Ready?" For every number, the experimenter prompted the child by saying, " $N$, what comes next?" The experimenter always began the task with 1 and provided feedback to the child if they were uncertain as to how they should respond. This first trial was excluded from analysis. The numbers in this task overlapped with the Unit Task (1-5), with each number tested twice in a pseudo-randomized order with no feedback.

\section{Results}

We first tested whether we replicated our findings from Experiment 1. We again found that children's CP knowledge explained unique variance beyond age in their Unit Task performance $\left(\chi^{2}(1)=4.19, p=.04\right)$, with significantly higher accuracy for CP-knowers $(\beta=0.47$, $p=.04 ; M=0.73)$ in comparison to subset-knowers $(M=0.57)$. Once again, however, subsetknowers were significantly above chance overall on the Unit Task (Wald $Z=2.50, p=.01$ ), and this effect was even stronger when the base addend was within their knower level (Wald $Z=$ $3.80, p=.0002)$. As in Experiment 1, follow-up analyses again showed evidence of item-specific 
knowledge for both CP and subset-knowers (Figure 4); while 1-knowers were at chance for all items (all $p \mathrm{~s}>.3$ ), 2-knowers were above chance for sets of $1(t(17)=3.34, p=.004)$. In contrast to Experiment 1, we found that 3-knowers were only above chance for sets of $1(t(7)=2.65, p=$ .03), which may be due to the small number of 3-knowers in this sample. We again found that, while CP-knowers were more accurate overall, this advantage was limited to sets of 1-3, with chance performance for sets of $4(t(27)=0.72, p=0.50)$ and $5(t(27)=1.22, p=0.23)$. Finally, we again found no relationship between Unit Task performance and Highest Count for subsetknowers $\left(\chi^{2}(1)=1.18, p=.28\right)$ or CP-knowers $\left(\chi^{2}(1)=1.21, p=.27\right)$.
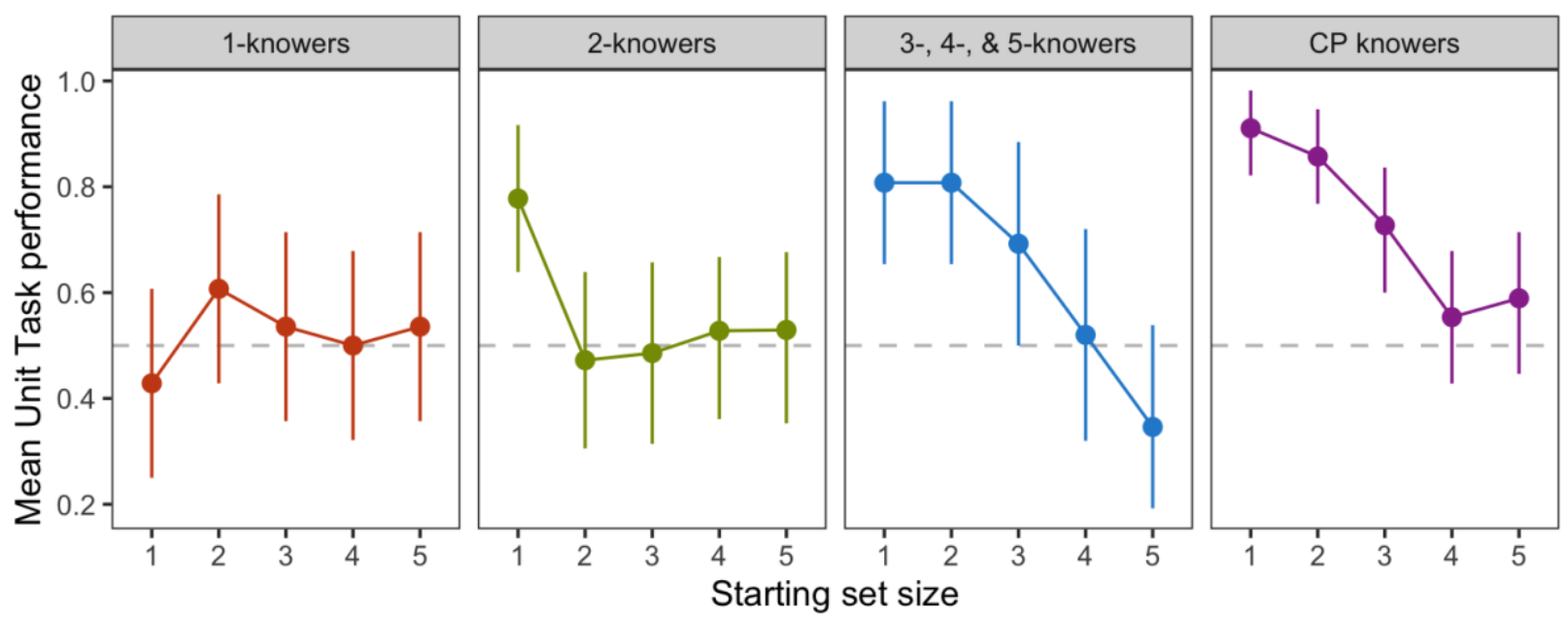

Figure 4. Mean accuracy on the Unit Task by set size and knower level. Error bars indicate $95 \%$ confidence intervals computed by nonparametric bootstrap, and dashed lines indicate chance performance (50\%).

To test whether CP and subset-knowers succeed in the Unit Task by reasoning about count list successor relations, we explored the relationship between performance on the Next Number and Unit Tasks. If children recruit knowledge of the count list's ordinal structure in the Unit Task (e.g., by reasoning that adding one item to a set of two should be labeled by "three," the number label one later in the count list), we should find either that children perform better on Next Number, or exhibit no difference between the two tasks. 


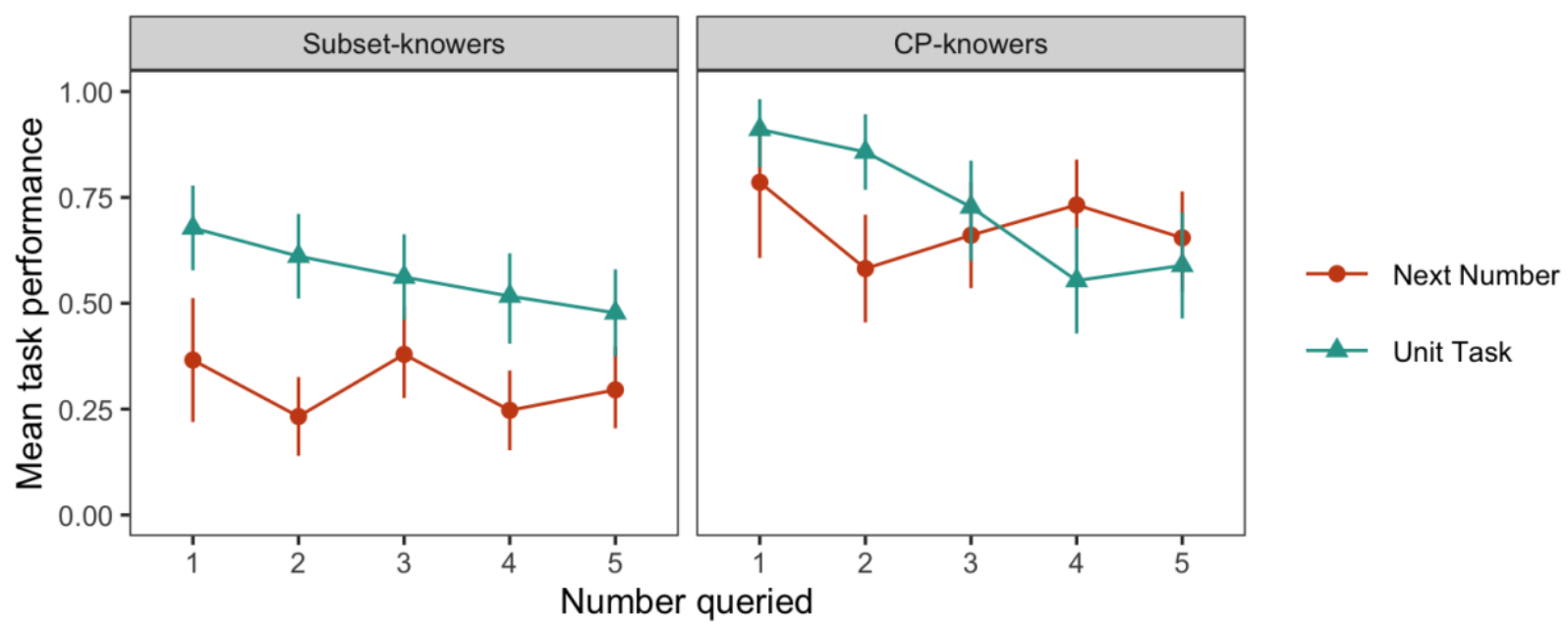

Figure 5. Mean accuracy on the Unit and Next Number tasks for each number queried, grouped by knower level. Error bars indicate $95 \%$ confidence intervals computed by nonparametric bootstrap.

We examined this separately in CP and subset-knowers with a GLMM ${ }^{4}$ predicting a correct response on the Unit Task and Next Number and controlling for age. Consistent with an Associative Mapping account, for subset knowers this model revealed significantly lower accuracy on Next Number in comparison to the Unit Task $(\beta=-1.21, p<.0001 ; M=0.30)$, with no advantage for known numbers, unlike in the Unit Task $(\beta=-1.48, p<.0001 ; M=0.32)$, as shown in Figure 5. This difference suggests that subset-knowers' ability to label Unit Task addition events is likely not due to reasoning about an isomorphism between count list successor relations and set operations. However, consistent with previous findings (Schneider et al., under review), in a separate GLMM we found no difference in accuracy between these two tasks for CP-knowers $(\beta=0.33, p=.11)$. While CP-knowers' equivalent performance on these tasks is compatible with Structure Mapping, given this group's chance performance for sets of 4 and 5, and the small size of this effect $(d=.26)$ it is possible that such a mapping is fragile at this point in development and may not be deployed by all children. Finally, we also conducted a follow up analysis to test whether children's performance on the Next Number task predicted Unit Task

\footnotetext{
${ }^{4}$ Model specifications: Correct $\sim$ Task (Unit Task/Next Number) + Age $+(1 \mid$ Participant).
} 
success for the same item; that is, whether children who could say that "three" came after "two" were also more likely to succeed for sets of 2 in the Unit Task. We conducted this analysis separately in subset- and CP-knowers, using a GLMM to predict Unit Task accuracy from Next Number performance for the same item, controlling for age, and with a random effect of participant. ${ }^{5}$ Compatible with the hypothesis that neither subset- nor CP-knowers were using count list knowledge to succeed on the Unit Task, we did not find that Next Number performance explained additional variance beyond age in predicting Unit Task success for both subset-knowers $\left(\chi^{2}(1)=2.57, p=.11\right)$ and CP-knowers $\left(\chi^{2}(1)=1.57, p=.21\right)$.

\section{Discussion}

In Experiment 2 we sought to replicate the results of Experiment 1 and to further explore the mechanisms through which children map the results of set operations in the Unit Task to number labels. One possibility is that children accomplish this through a Structure Mapping between the count list and cardinality; specifically, that adding +1 to a set corresponds to counting-up one item in the count list. Such a mechanism hinges on knowledge of relations between number words in the count list; in particular, the ability to count up from a given number to identify the label of its successor. In contrast, the Associative Mapping hypothesis holds that children identify the labels of sets by observing the sets and set operations involved in the Unit Task directly, then mapping known numeral labels onto these magnitude representations. Unlike a Structure Mapping account, this Associative Mapping mechanism can be deployed independently of any knowledge of count list successor relations.

We found two main results. First, compatible with Experiment 1, we again found that subset-knowers succeeded in the Unit Task for small sets within their knower level, and

\footnotetext{
${ }^{5}$ Model specifications: Unit Task correct $\sim$ Next Number correct + Age $+(1 \mid$ Participant $)$
} 
consequently that item-specific success on this task emerges before CP acquisition. Second, we found that subset-knowers' item-specific Unit Task success was unrelated to their performance on Next Number; that is, e.g., a three-knower could correctly infer that adding one item to a set of two should be labeled by "three," but was generally unable to answer, "What number comes after $t w o$ ?" Together, these results suggest that children's ability to infer the label of Unit Task addition events does not initially involve count list knowledge in the sense implied by Structural Mapping. Instead, their success is initially facilitated by associations between specific number words and small sets.

However, our data are also consistent with the idea that CP-knowers use Structure Mapping to succeed at the Unit Task: although CP-knowers' Next Number performance did not predict Unit Task accuracy, we found no difference in mean performance between these two tasks, and greater Unit Task accuracy by CP-knowers in comparison to subset-knowers. These findings leave open the possibility that CP-knowers leverage their greater knowledge of count list successor relations to infer the labels of Unit Task set operations. However, CP-knowers' chance performance for the largest numbers queried in the Unit Task (i.e., 4 and 5) suggests that if CP-knowers do deploy such a Structure Mapping, they do not do so uniformly, even when they have adequate knowledge of count list successor relations for the cardinalities involved. While we found a relationship between Next Number and the Unit Task in CP-knowers, however, it is possible that subset-knowers may fail Next Number for reasons unrelated to number knowledge. Thus, while our results may indicate some support for Structure Mapping in CP-knowers, but not subset-knowers, future studies may find evidence of this mechanism in subset-knowers with a more sensitive measure. 
In sum, we conclude that early in number word learning, children's limited Unit Task success is probably not driven by reasoning about count list successor relations (i.e., Structure Mapping). Instead, children's success appears to be supported by direct associations between set representations and verbal labels.

\section{General Discussion}

One of the most powerful tools a learner wields is the ability to use relational structures within one domain to discover and generalize abstract concepts in another. To accomplish this, the learner must first acquire the relevant structures and then recognize the commonalities over which they can posit a Structure Mapping (Gentner, 2010). Also, they must extend this mapping beyond a few isolated examples to induce a generalized principle. Here, we explored how such Structure Mapping processes emerge in children within a paradigmatic case study of relational learning: symbolic number acquisition. While children begin reciting the count list early in development, it takes them years to become competent counters. To do so, they must ultimately learn that counting up 1 word in the count list corresponds to adding 1 item to a set, and vice versa - a Structure Mapping sometimes described as implicit successor function knowledge (Sarnecka \& Carey, 2008). By some accounts (Carey, 2004; Gentner, 2010), this mapping allows children to transition from subset-knowers to CP-knowers: In contrast to subset-knowers, who are limited to labeling a few sets with known number words (i.e., Associative Mapping), CPknowers can construct and label an indefinite number of new sets by simply counting up in the count list, and adding one item for every additional numeral.

However, recent empirical work has questioned whether $\mathrm{CP}$ acquisition implicates Structure Mapping by showing that many CP-knowers appear to lack a strong, generalized understanding of how counting up is related to changes in cardinality. For example, although 
some CP-knowers can label small sets after a Unit Task addition event (e.g., judging that a set of "four" becomes "five" after adding 1 item), many fail this task even for small numbers, and those who succeed often struggle with only modestly larger numbers (Cheung et al., 2017; Davidson et al., 2012; Spaepen et al., 2018). Such evidence challenges the idea that Structure Mapping underlies children's transition to becoming CP-knowers, and suggests that such knowledge may emerge much later in acquisition - as late as 6 years of age (Cheung et al., 2017). These studies raise the possibility that young CP-knowers' limited Unit Task success relies not on Structure Mapping, but instead on a simpler process of Associative Mapping, which does not involve reasoning about successor relations in the count list. If CP-knowers use Associative Mapping, then previously reported differences between them and subset-knowers on measures that ostensibly target the outputs of Structure Mapping (such as the Unit Task) may not be qualitative in nature, but instead due to the amount of exposure to specific numbers.

In line with this hypothesis, here we find that even subset-knowers perform well on the Unit Task when tested with small numbers within their known range. In two experiments, Unit Task success preceded CP acquisition, and was related to familiarity with specific number words: subset-knowers performed significantly better than chance, and did so specifically for known numbers words. In Experiment 2 we found evidence that this item-specific Unit Task success is not initially supported by a Structure Mapping that exploits knowledge of successor relations in the count list: Next Number performance, which measures knowledge of these successor relations, was unrelated to Unit Task performance in subset-knowers, and was only weakly related in CP-knowers. Additionally, neither subset- nor CP-knowers' performance on the Next Number task predicted their Unit Task success. However, Unit Task performance was related to knowledge of number words, such that, e.g., two-knowers could perform operations 
that one-knowers could not, suggesting that both individual differences and cross-linguistic differences in the rate of early number word learning could impact children's performance on the Unit Task (e.g., lending an advantage to children learning languages with rich number marking, such as Arabic or Slovenian; Almoammer, Sullivan, Donlan, Marušič, O’Donnell, \& Barner, 2013; Marušič et al., 2016). Together, these findings indicate that, rather than labeling a Unit Task set operation by recognizing that adding one item to a set requires counting up a corresponding number in the count list, children may initially solve this task through directly mapping known number words to set representations. These findings are compatible with the hypothesis that such item-specific mappings are based on an Associative, rather than Structure, Mapping mechanism, and therefore, that the creation of such a Structure Mapping may indeed be gradual, and not the basis for $\mathrm{CP}$ acquisition.

Although CP-knowers generally outperformed subset-knowers on the Unit Task and Next Number, these results do not provide strong evidence for the use of a Structure Mapping, even in these children. First, the ability to reason about relations between numbers - as required by the Next Number task - is necessary for a Structure Mapping, but not sufficient. Also needed is the actual mapping from these relations between numbers to set operations. But more important, in both Experiments 1 and 2, CP-knowers' Unit Task advantage was limited to sets of 1-3, with either lower or at-chance performance for sets of 4 and 5. Thus, many CP-knowers in our sample failed both the Unit Task and the Next Number Task for numbers beyond the subsetknower stage, despite being able to count and generate larger sets in the Give-N task. This suggests that classification as $\mathrm{CP}$-knowers on the Give-N task — and in particular the ability to give sets of 5 and 6 - could not have been driven by a Structure Mapping, and, therefore, such a mapping cannot explain the transition to the $\mathrm{CP}$ stage. 
Importantly, this lower performance for larger sets persisted even when CP-knowers had adequate knowledge of relations between these number words, as indicated by their performance on Next Number. Together, these results suggest that while young CP-knowers have greater knowledge of count list successor relations, they do not initially show evidence of a Structure Mapping that extends beyond the small number range. $\mathrm{CP}$-knowers may deploy a Structure Mapping for only small numbers, or may not use a Structure Mapping at all, despite having preliminary knowledge of how small number words are related. One possibility is that this failure reflects a general inability to use Structure Mappings at this early stage of learning, whether for large numbers or for small ones. In this scenario, CP-knowers' performance might depend exclusively on Associative Mappings, both for small numbers (1-3) and for slightly larger ones, whether via the use of slightly noisier associations to the Approximate Number System, or via the use of chunking strategies that extend the range of parallel individuation (Feigenson \& Halberda, 2004; Moher, Tuerk, \& Feigenson, 2012). Finally, it remains possible that CP-knowers attempt to use Structure Mapping for larger numbers but fail due to either limited count list knowledge, or to problems coordinating count list knowledge and the set operations involved in the Unit Task. Future work should explore the mechanisms underlying CP-knowers' item-based success for larger numbers.

These results provide key data on an outstanding question in children's acquisition of successor function knowledge, namely why otherwise competent counters demonstrate a fragmented understanding of this principle for years. While initially theorized to be acquired in conjunction with the CP (Carey, 2004; Sarnecka \& Carey, 2008), recent evidence has established a more extended timeline suggesting generalized successor knowledge is not fully acquired until around age 6 (Cheung, et al., 2017; Schneider et al., 2020). Our results suggest that at least one 
factor limiting early successor knowledge is that it is initially not relational in nature. That is, some children appear to pass the Unit Task by mapping the results of set operations directly to known number labels, only later discovering the Structure Mapping between those operations and counting. Such a pattern is consistent with the hypothesis that acquisition of the CP marks not a conceptual shift in children's understanding of counting and cardinality, but rather simply learning another procedure attached to counting (Barner, 2018; Davidson et al., 2012).

One important conclusion of this study is that children begin to use item-based Associative Mappings to compute addition operations early in development, raising the question of whether this ability plays a role in the construction of a Structure Mapping. One surprising, but informative, aspect of our findings is that young children succeed at what is arguably the most difficult precursor to a Structure Mapping between counting and cardinality. Specifically, even subset-knowers represented small sets under occlusion, updated them after addition events, and associated them with labels. This ability represents one half of the Structure Mapping; the other half is the ability to identify the successors of number words - e.g., that "five" comes after "four." When combined, these two abilities allow children to infer the labels of addition operations by counting up in the count list.

Given this, our data suggest that even subset-knowers are well on their way to acquiring the conceptual component of the Structure Mapping, and are missing only knowledge of (1) relations between numbers, and (2) how these relations correspond to set operations. Our data suggest that knowledge of relations between numbers, as tested by the Next Number task, begins to emerge sometime after children become $\mathrm{CP}$-knowers. Previous data suggest that this learning process continues for several years, until children acquire a morphosyntactic rule that allows them to combine the numbers from 1-9 with the decades from 20-90 to productively generate 
ever larger numbers, and perhaps to support the inference that, because numbers are generated by a rule, they may continue infinitely (Cheung et al., 2017; Chu et al., 2020; Schneider et al., 2020). Given this timeline for children's acquisition of relations between numbers, the ability to link this knowledge to set operations must also emerge gradually after $\mathrm{CP}$ acquisition. This suggests that the procedures children acquire at the $\mathrm{CP}$ stage may create new conditions for learning, perhaps by allowing children to repeatedly notice how counting up is related to adding objects to sets. On this hypothesis, procedures may provide a workspace for conceptual discovery. 


\section{References}

Almoammer, A., Sullivan, J., Donlan, C., Marušič, F., O’Donnell, T., \& Barner, D. (2013). Grammatical morphology as a source of early number word meanings. Proceedings of the National Academy of Sciences, 110(46), 18448-18453.

Aristotle, \& Ross, W. D. (1981). Aristotle's Metaphysics. Oxford, England: Clarendon Press.

Bates, D., Maechler, M., Bolker, B., Walker, S. (2015). Fitting linear mixed-effects models using 'Ime4'. Journal of Statistical Software, 67(1), 1-48.

Cantrell, L., \& Smith, L. B. (2013). Open questions and a proposal: A critical review of the evidence on infant numerical abilities. Cognition, 128(3), 331-352. https://doi.org/10.1016/j.cognition.2013.04.008

Carey, S. (2004). Bootstrapping \& the origin of concepts. Daedalus, 133(1), 59-68. https://doi.org/10.1162/001152604772746701

Carey, S. (2009). Oxford series in cognitive development: Vol. 3. The origin of concepts. Oxford University Press.

Cheung, P., Rubenson, M., \& Barner, D. (2017). To infinity and beyond: Children generalize the successor function to all possible numbers years after learning to count. Cognitive Psychology, 92, 22-36. https://doi.org/10.1016/j.cogpsych.2016.11.002

Chu, J., Cheung, P., Schneider, R. M., Sullivan, J., \& Barner, D. (2020). Counting to Infinity: Does learning the syntax of the count list predict knowledge that numbers are infinite?. Cognitive Science 44 (8). https://doi.org/10.31234/osf.io/5c6pb

Davidson, K., Eng, K., \& Barner, D. (2012). Does learning to count involve a semantic induction?. Cognition, 123(1), 162-173. https://doi.org/10.1016/j.cognition.2011.12.013 
Feigenson, L., Carey, S., \& Hauser, M. (2002). The representations underlying infants' choice of more: Object files versus analog magnitudes. Psychological Science, 13(2), 150-156. https://doi.org/10.1111/1467-9280.00427

Feigenson, L., \& Halberda, J. (2004). Infants chunk object arrays into sets of individuals. Cognition, 91(2), 173-190. https://doi.org/10.1016/j.cognition.2003.09.003

Fuson, K. C. (1988). Children's counting and concepts of number. Springer Science \& Business Media.

Gelman, R., \& Gallistel, C. R. (1978). The Child's Understanding of Number. Cambridge, MA Harvard University Press.

Gentner, D. (2010). Bootstrapping the mind: Analogical processes and symbol systems. Cognitive Science, 34(5), 752-775. https://doi.org/10.1111/j.1551-6709.2010.01114.x

Gentner, D., Brem, S., Ferguson, R., \& Wolff, P. (1997). Analogy and creativity in the works of Johannes Kepler. In T. B. Ward, S. M. Smith, \& J. Vaid (Eds.), Creative thought: An investigation of conceptual structures and processes (p. 403-459). American Psychological Association. https://doi.org/10.1037/10227-016

Hallyn, F. (2000). Atoms and letters. In Metaphor and analogy in the sciences (pp. 53-69). Springer, Dordrecht.

Hartnett, P., \& Gelman, R. (1998). Early understandings of numbers: Paths or barriers to the construction of new understandings?. Learning and Instruction, 8(4), 341-374. https://doi.org/10.1016/S0959-4752(97)00026-1

Hughes, M. (1981). Can preschool children add and subtract?. Educational Psychology, 1(3), 207-219. https://doi.org/10.1080/0144341810010301 
Huttenlocher, J., Jordan, N. C., \& Levine, S. C. (1994). A mental model for early arithmetic. Journal of Experimental Psychology: General, 123(3), 284. https://doi.org/10.1037/00963445.123 .3 .284

Lefevre, J. A., Clarke, T., \& Stringer, A. P. (2002). Influences of language and parental involvement on the development of counting skills: Comparisons of French-and Englishspeaking Canadian children. Early Child Development and Care, 172(3), 283-300. https://doi.org/10.1080/03004430212127

Marchand, E., \& Barner, D. (2018). Analogical mapping in numerical development. In Language and Culture in Mathematical Cognition (pp. 31-47). Academic Press. https://www.sciencedirect.com/science/article/pii/B978012812574800002X

Moher, M., Tuerk, A. S., \& Feigenson, L. (2012). Seven-month-old infants chunk items in memory. Journal of experimental child psychology, 112(4), 361-377. https://doi.org/10.1016/j.jecp.2012.03.007

Peano, G. (1889). Arithmetices principia: nova methodo. Fratres Bocca.

Sarnecka, B. W., \& Carey, S. (2008). How counting represents number: What children must learn and when they learn it. Cognition, 108(3), 662-674. https://doi.org/10.1016/j.cognition.2008.05.007

Schneider, R., Sullivan, J., Guo, K., Barner, D. (under review). What counts? Sources of knowledge in children's acquisition of the successor function.

Schneider, R. M., Sullivan, J., Marušič, F., Biswas, P., Mišmaš, P., Plesničar, V., \& Barner, D. (2020). Do children use language structure to discover the recursive rules of counting?. Cognitive Psychology, 117, 201-263. https://doi.org/10.1016/j.cogpsych.2019.101263 
Siegler, R. S., \& Robinson, M. (1982). The development of numerical understandings. In Advances in Child Development and Behavior (Vol. 16, pp. 241-312). JAI. https://doi.org/10.1016/S0065-2407(08)60072-5

Spaepen, E., Gunderson, E. A., Gibson, D., Goldin-Meadow, S., \& Levine, S. C. (2018). Meaning before order: Cardinal principle knowledge predicts improvement in understanding the successor principle and exact ordering. Cognition, 180, 59-81. https://doi.org/10.1016/j.cognition.2018.06.012

Sullivan, J., \& Barner, D. (2014). Inference and association in children's early numerical estimation. Child development, 85(4), 1740-1755. https://doi.org/10.1111/cdev.12211

Wynn, K. (1990). Children's understanding of counting. Cognition, 36(2), 155-193. https://doi.org/10.1016/0010-0277(90)90003-3

Wynn, K. (1992a). Addition and subtraction by human infants. Nature, 358(6389), 749-750. https://doi.org/10.1038/358749a0

Wynn, K. (1992b). Children's acquisition of the number words and the counting system. Cognitive Psychology, 24(2), 220-251. https://doi.org/10.1016/0010-0285(92)90008-P

Xu, F. (2019) Towards a rational constructivist theory of cognitive development. Psychological Review, 126, 841-864. https://doi.org/10.1037/rev0000153 